\title{
La poche ciliaire: fruit des liaisons du centrosome avec le trafic vésiculaire
}

des mécanismes complexes et conservés au cours de l'évolution, qui aboutissent à l'ancrage du corps basal aux membranes cellulaires. Des données anciennes de la littérature ont suggéré que, dans de nombreux types cellulaires, cet ancrage se fait dans le cytoplasme et que le cil se forme au sein d'une vésicule avant d'être exporté vers la membrane plasmique. Cette voie intracellulaire, récemment caractérisée sur le plan moléculaire, aboutit à la formation d'un domaine membranaire à la base des cils, la poche ciliaire, dont la caractérisation récente a permis de montrer son implication dans le trafic vésiculaire et la signalisation. <

Les cils et flagelles sont des structures très conservées au cours de l'évolution qui ont des rôles essentiels chez les organismes ciliés où ils exercent des fonctions motrices et/ou sensorielles [1]. Les cils primaires sont une sous-population de cils, présents en copie unique à la surface de la plupart des types cellulaires chez les vertébrés. Ils sont caractérisés par la nature de leur corps basal et par la structure de leur axonème, constitué de neuf doublets de microtubules périphériques (cils $9+0$; Figure IA) [36] $(\rightarrow)$.

$\rightarrow$ Voir la Synthèse de C. Fort et P. Bastin, page 955 de ce numéro

Bien que leur fonction sensorielle ait été immédiatement suggérée en raison de leur organisation similaire à celle du cil connecteur des photorécepteurs [2], leur rôle exact est resté mystérieux pendant 40 ans, de leur première caractérisation en microscopie électronique en 1961 [2] à la mise en évidence du lien entre ces structures et les maladies kystiques rénales par le groupe de Joël Rosembaum en 2001 [3]. De très nombreux travaux ont, depuis, démontré le rôle central des cils primaires dans des fonctions sensorielles (mécaniques, odeurs, lumière) et dans le contrôle de

Cet article fait partie du numéro thématique de médecine/sciences intitulé « Cils primaires et ciliopathies $\gg\left(\mathrm{m} / \mathrm{s} \mathrm{n}^{\circ} 11\right.$, vol. 30 , novembre 2014$)$.

\section{Alexandre Benmerah}

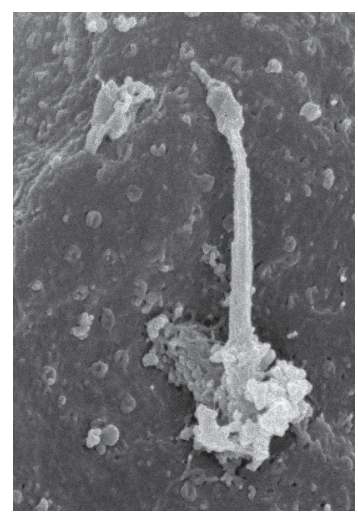

Inserm UMR 1163,

laboratoire des maladies rénales héréditaires, Paris, France ; Université Paris DescartesSorbonne Paris Cité, institut Imagine, 24, boulevard du Montparnasse, 75015 Paris, France. alexandre.benmerah@inserm.fr nombreuses voies de signalisation qui jouent un rôle clé dans le développement [37] $(\rightarrow)$ et dans l'homéostasie tissulaire (signalisation Hedgehog et Wnt/PCP [planar cell polarity]) [38] $(\rightarrow)$.

Les dysfonctionnements des cils primaires sont donc responsables de maladies complexes associant une rétinite pigmentaire, des atteintes rénales (néphronophtise ou polykystose), une obésité et/ou des atteintes du système nerveux central (agénésie du cervelet). La présence d'une ou plusieurs de ces manifestions a permis de définir plusieurs syndromes regroupés sous le terme de «ciliopathies » [39-4l] $(\rightarrow)$.

$(\rightarrow)$ Voir la Synthèse de N. Biguet et S.M. Meilhac, page 996 de ce numéro

$(\rightarrow)$ Voir la Synthèse de J. Ezan et M. Montcouquiol, page 1004 de ce numéro

$(\rightarrow)$ Voir les Synthèses de M. Paces-Fessy ; de R. Bachmann-Gagescu ; de $K$. Chennen et al., pages 1024, 1011 et 1034 de ce numéro Les cils sont assemblés à partir d'un centriole qui prend alors le nom de corps basal; celui-ci peut correspondre au centriole parental du centrosome (cil primaire) ou à des centrioles amplifiés (cils mobiles). Ces centrioles, le centriole père (mother centriole) pour le cil primaire, vont s'ancrer aux membranes cellulaires par leurs appendices distaux. Les appendices distaux sont des structures spécifiques des centrioles pères, comme les appendices subdistaux ou pieds basaux (pour les cils mobiles), ces derniers étant impliqués dans l'ancrage des microtubules (Figure 1B). Une fois les corps basaux ancrés, la formation de l'axonème est assurée par une machinerie spécifique, I'IFT (intraflagellar transport) et le BBSome (Bardet-Biedl syndrome proteins), qui va permettre le transport des constituants de l'axonème et de la membrane ciliaire vers le compartiment ciliaire [36] $(\rightarrow)$.

$(\rightarrow)$ Voir la Synthèse de C. Fort et P. Bastin, page 955 de ce numéro 

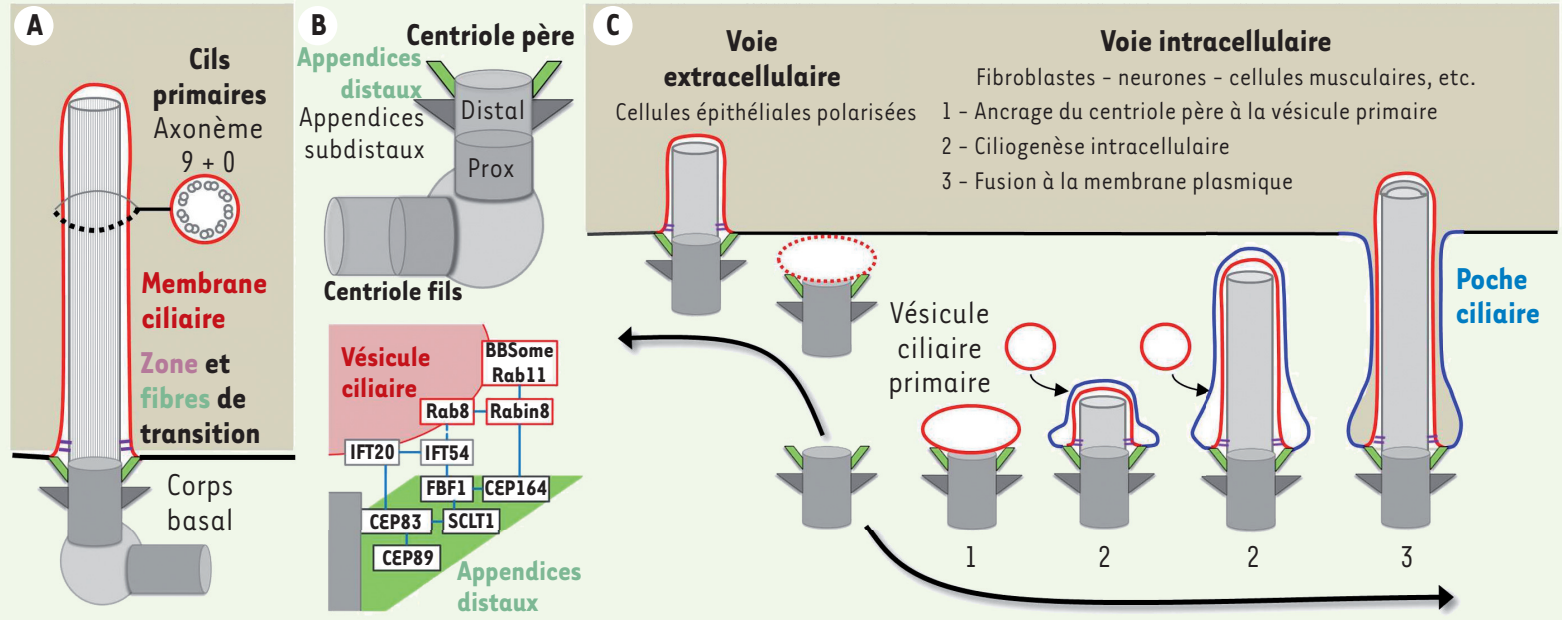

Figure 1. Ciliogenèse primaire. A. Les cils primaires sont constitués d'un corps basal (centrosome) et d'un axonème présentant neuf doublets de microtubules. Le centriole père est ancré à la membrane via ses appendices distaux ( $A D$ ) ou fibres de transition (vert) qui forment avec la zone de transition (violet) une barrière limitant les échanges cil/cytoplasme. B. Organisation du centrosome et des appendices distaux (vert) et leurs interactions avec des constituants de la membrane de la vésicule ciliaire (rouge) et les IFT (IFT20/54; gris). Les différentes protéines constituant les appendices distaux (PAD) ont été indiquées : CEP83/CCDC41, CEP89/CEP123/CCDC123, SCLT1, FBF1 et CEP164. Prox. : proximal. C. Deux voies distinctes permettent la formation des cils primaires. Dans la voie « extracellulaire », le corps basal s'ancre à la membrane cellulaire et le cil pousse directement vers le milieu extracellulaire. Dans la voie «intracellulaire», le corps basal s'ancre à une vésicule (vésicule ciliaire, rouge) dans le cytoplasme, le cil croît au sein de cette vésicule et atteint le milieu extracellulaire lorsque la vésicule fusionne avec la membrane plasmique. Le relargage incomplet du cil après la fusion de la vésicule à la membrane donne naissance à un domaine membranaire à la base du cil, la poche ciliaire.

La formation de tous les types de cils démarre donc par l'interaction du futur corps basal avec les membranes cellulaires. Le modèle a longtemps été présenté comme un ancrage direct des centrioles à la membrane plasmique. Cependant, S. Sorokin avait proposé dès 1962 un modèle plus complexe pour la ciliogenèse des cils primaires (ciliogenèse primaire). Dans ce processus détaillé ci-après, le cil se forme au sein d'une vésicule dans le cytoplasme et est ensuite libéré à la surface cellulaire par fusion de la vésicule avec la membrane plasmique [4]. De façon intéressante, des travaux récents ont caractérisé la présence d'un domaine membranaire à la base des cils, la poche ciliaire, qui serait la conséquence directe de cette voie intracellulaire de formation des cils [5]. Le but de cette revue est de décrire ces connexions entre centriole et trafic vésiculaire à la base de la formation des cils, et les fonctions de la poche ciliaire dans le trafic vésiculaire associé au cil.

\section{Ciliogenèse, interaction intime du centriole avec les membranes cellulaires}

Chez les mammifères, les différentes étapes de formation des cils ont été décrites par S. Sorokin en 1968 [4], grâce à une formidable étude en microscopie électronique. Cet auteur donnera ainsi le nom de cil primaire à ces cils uniques (un seul par cellule) qui présentent un corps basal (centriole père du centrosome) et un axonème dépourvu de doublet central $(9+0)$. Le terme primaire n'est pas à prendre dans le sens péjoratif, contrairement au terme « vestigial » ou « rudimentaire » que $\mathrm{S}$. Sorokin utilise lui-même dans sa première étude [6], mais il souligne le fait que ces cils apparaissent en premier au cours du développement et sont présents à la surface des cellules épithéliales bronchiques avant la formation des cils mobiles.

S. Sorokin propose donc deux voies distinctes de formation des cils primaires en fonction des types cellulaires. Dans la plupart des cellules (fibroblastes, myocytes), le cil primaire se forme au sein du cytoplasme (cycle intracellulaire, Figure 1C). Au cours de ce processus, un des deux centrioles du centrosome (centriole père) s'associe par son extrémité distale à une vésicule cytoplasmique (vésicule ciliaire primaire $\mathrm{Cl}$ ) pouvant provenir du Golgi. L'axonème se forme au sein de cette vésicule qui s'allonge grâce à un apport de membrane par fusion avec d'autres vésicules (vésicule (2). Le cil primaire ainsi préassemblé est libéré à la surface cellulaire par fusion de la vésicule ciliaire avec la membrane plasmique (vésicule (3). Dans les cellules épithéliales, S. Sorokin n'avait pas pu observer d'intermédiaire vésiculaire; il a donc proposé qu'un des deux centrioles 
s'ancre directement à la membrane apicale à partir duquel le cil s'assemblerait à la surface cellulaire.

Alors que des travaux récents ont confirmé les hypothèses de $S$. Sorokin concernant la voie intracellulaire (voir ci-après), très peu d'études se sont intéressées aux étapes précoces de formation des cils primaires dans les cellules épithéliales polarisées. S. Sorokin luimême n'excluait pas la possibilité d'un intermédiaire vésiculaire lors de la ciliogenèse primaire dans ces cellules [4]. De façon intéressante, dans le modèle des cellules multiciliées de l'épiderme de l'embryon de xénope (cils mobiles), les corps basaux s'associent à une vésicule avant leur ancrage à la membrane apicale, sans phase de croissance intracellulaire de l'axonème [7]. Un tel mécanisme pourrait donc représenter un modèle alternatif pour la formation des cils primaires dans les cellules épithéliales.

\section{Cinquante ans après, les bases moléculaires de l'interaction centriole/vésicule ciliaire}

Plusieurs études ont récemment caractérisé d'un point de vue moléculaire les mécanismes décrits par S. Sorokin il y a plus de 45 ans. La plupart de ces études ont été réalisées dans les cellules RPEl (retinal pigment epithelial) qui sont le modèle cellulaire le plus couramment utilisé pour étudier la ciliogenèse primaire in vitro. Dans ces cellules, la présence d'une poche ciliaire à la base des cils suggérait que ceuxci se forment par la voie intracellulaire. Ces études ont confirmé l'ancrage d'une vésicule au centriole père via les appendices distaux, des structures des centrosomes qui n'ont été mises en évidences qu'après le travail de S. Sorokin [8].

\section{Les appendices distaux}

Les appendices distaux émanent de l'extrémité distale du centriole père et leur formation est sous le contrôle de C2CD3 (C2 calciumdependent domain contaning 3) [9] et d'ODF2 (outer dense fibre protein 2), qui contrôle aussi la formation des appendices subdistaux [10]. Les appendices distaux forment une couronne qui interagit avec la membrane cellulaire et définissent les fibres de transition à la base du futur cil (Figure IA) [8, 10]. Ces fibres de transition et la zone de transition à la base de l'axonème forment une structure agissant comme un filtre moléculaire qui contrôle le passage des protéines vers ou en dehors du cil. La composition des appendices distaux vient d'être récemment déterminée [11]: les protéines qui les constituent (PAD) (Figure IB) sont requises pour la formation des cils primaires [11-15]. CEP83 (centrosomal protein of 83-kDa)/CCDC41 (coiled-coil domain containing 41) joue un rôle central car elle est requise pour l'ancrage de toutes les autres protéines des appendices distaux au centriole père [11]. En l'absence de CEP83, le centriole père est incapable de s'ancrer aux membranes cellulaires, et il est retrouvé libre dans le cytoplasme [11, 13]. Des observations similaires avaient été faites pour CEP164, une des premières protéines des appendices distaux identifiées [12]. Des mutations du gène codant pour CEPl64 sont présentes dans des formes de ciliopathies associant rétinite pigmentaire, néphro- nophtise et défauts du système nerveux central [16]. Des mutations de SCLTI (sodium channel and clathrin linker 1) ont été identifiées chez un patient atteint d'un syndrome oro-facio-digital de type IX [17]. Enfin, nos résultats récents indiquent que des mutations de CEP83 sont responsables de formes précoces de néphronophtise associées à une hydrocéphalie et des déficits cognitifs [18].

\section{La vésicule ciliaire primaire}

Deux protéines de la future membrane ciliaire ont pu être localisées au niveau de la vésicule ciliaire, les protéines Smo (Smoothened) et Rab8.

- Rab8 est une GTPase de la famille Rab qui est impliquée dans la voie de sécrétion du Golgi vers les endosomes et/ou vers la membrane plasmique [19]. Récemment, il a été montré que Rab8 interagit avec le BBSome au cours de la ciliogenèse, et joue un rôle important dans la croissance de la membrane ciliaire [20]. Sa localisation proche du centrosome au cours des étapes très précoces de la ciliogenèse indique sa présence sur la vésicule ciliaire. Son recrutement et son activation seraient sous le contrôle de CEP164 via son interaction avec Rabin8 [21], un facteur d'échange de Rab8 qui est associé à des vésicules contenant Rabll (endosomes de recyclage), elle-même requise pour la ciliogenèse [22]. Rabll et CEP164 participeraient ainsi à l'activation coordonnée de Rabin8 qui, à son tour, activerait Rab8 à la membrane de la vésicule ciliaire au contact des appendices distaux. Cette activation coordonnée de Rab8 sur la membrane de la vésicule ciliaire contrôlerait l'élongation de cette membrane par un mécanisme qui reste à déterminer.

- Smo est un acteur clé de la voie Hedgehog ; il est recruté à la membrane ciliaire au cours de l'activation de cette voie, mais y est présent de façon constitutive quand il est surexprimé. Dans ce dernier cas, la présence de Smo à la vésicule ciliaire $[13,23]$ indique que la vésicule contient déjà des cargos destinés à la membrane ciliaire. Ceci suggère qu'il existe une étape de tri, à partir de la voie de biosynthèse ou de compartiments endosomaux, qui permet d'incorporer les protéines membranaires ciliaires dans des vésicules en cours d'adressage vers le cil. Ce tri pourrait s'opérer au niveau du Golgi où réside IFT20 (intraflagellar transport 20), une sous-unité du complexe IFT-B qui a été impliquée dans le trafic vésiculaire des protéines membranaires ciliaires et du récepteur $T$ des lymphocytes [24, 25]. De plus, IFT20 interagit avec CEP83, qui est requise pour son recrutement au centrosome [13]. IFT20 interagit également avec IFT54 au sein du complexe IFT-B, mais IFT54 lie aussi FBFl (Fas binding factor 1) [15], une 

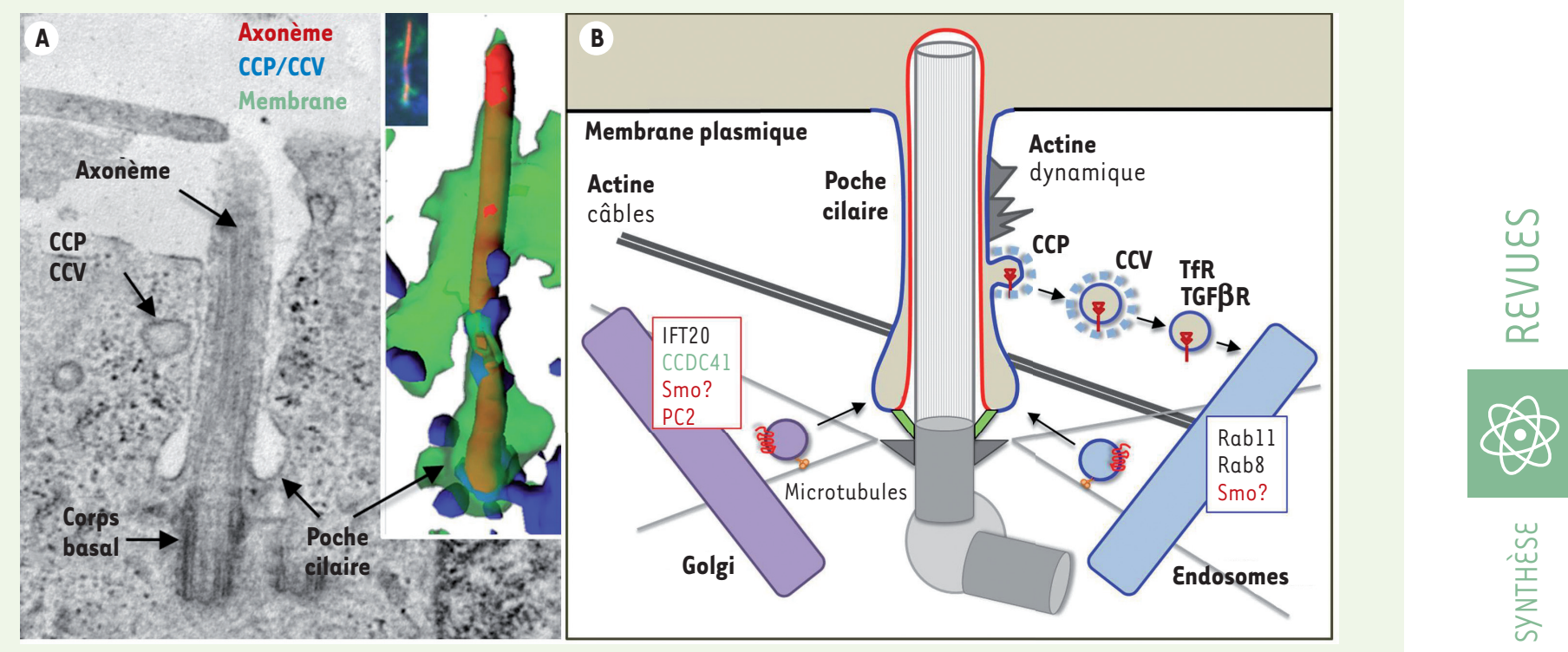

Figure 2. La poche ciliaire. A. Image de microscopie électronique montrant une coupe longitudinale d'un cil primaire (cellules RPEl) enraciné dans le cytoplasme et dont la région proximale est entourée d'une invagination de la membrane plasmique, la poche ciliaire, d'où bourgeonnent des puits (CCP) et vésicules recouverts de clathrine (CCV). En insert, sur la droite, une reconstruction 3D d'images de fluorescence obtenues à partir du même type cellulaire avec, en vert, un marqueur de la poche ciliaire, en continuité avec la membrane plasmique qui entoure la région proximale du cil (rouge) et sur laquelle bourgeonnent des puits recouverts de clathrine (bleu). B. Représentation schématique de la poche ciliaire, de ses fonctions dans l'endocytose dépendante de la clathrine et de ses connexions avec le trafic vésiculaire (Golgi/endosomes) et le cytosquelette d'actine. TfR : récepteur de la transferrine.

autre PAD [11]. IFT20 pourrait donc être impliquée, à la fois dans le bourgeonnement de précurseurs de la vésicule ciliaire à partir du Golgi, et dans l'adressage de cette vésicule aux appendices distaux. Des connexions entre IFT54 et Rab8 ont également été décrites [26], suggérant que les protéines des complexes IFT-B associées aux appendices distaux joueraient un rôle important dans le processus d'ancrage de la vésicule ciliaire et dans les phases précoces de la ciliogenèse, de façon indépendante de leur rôle classique dans l'IFT.

\section{Croissance intracellulaire et délivrance}

Après la phase initiale d'ancrage de la vésicule ciliaire, le cil poursuivrait une phase de croissance intracellulaire au cours de laquelle ont lieu l'élongation de l'axonème et la formation de la zone de transition. L'élongation de l'axonème au sein de la vésicule implique une croissance en parallèle de la membrane ciliaire et de la vésicule ciliaire dans laquelle le cil s'allonge, et nécessite donc un apport de membrane important (Figure 1C). Il a été rapporté que des vésicules contenant Smo s'agrègent autour du centriole au cours de la ciliogenèse $[13,23]$, en accord avec l'observation de $S$. Sorokin montrant l'arrimage de vésicules secondaires à la vésicule ciliaire [4]. Ces données indiquent que ces vésicules apporteraient, outre la membrane nécessaire, des constituants de la membrane ciliaire. L'origine et les mécanismes qui permettent l'adressage et l'arrimage de ces vésicules à la vésicule ciliaire restent inconnus.
Le relargage du cil dans le milieu extérieur implique l'arrimage, puis la fusion de la vésicule ciliaire à la membrane plasmique. Cette dernière étape n'a pas non plus été caractérisée. II est cependant tentant d'imaginer un rôle de l'exocyste, machinerie permettant l'ancrage et la fusion des vésicules d'exocytose avec la membrane plasmique. Certaines sous-unités de l'exocyste sont en effet requises pour la ciliogenèse (SEC10, SEC15) [27, 28]. De plus, dans les cellules multiciliées de l'épiderme de l'embryon de xénope, l'association des corps basaux à des vésicules exprimant SEC8 est requise pour leur ancrage à la membrane apicale [7].

Enfin, il est souvent indiqué que le corps basal «migre » vers la membrane plasmique. Si une telle assertion semble être tout à fait pertinente pour la formation des cils à la membrane apicale, on peut se poser la question de l'existence d'un tel mécanisme lorsque la ciliogenèse met en jeu la voie intracellulaire. $\varepsilon$ n effet, la distance noyau centrosome/corps basal semble constante, stable et finement régulée par des mécanismes permettant la cohésion entre noyau et centrosome [29]. II est donc possible d'imaginer que l'allongement progressif du cil favorise le déplacement de l'extrémité distale de la membrane de la vésicule ciliaire, jusqu'à permettre 
les étapes d'arrimage et de fusion à la membrane plasmique, sans déplacement actif du corps basal par rapport au noyau.

\section{La poche ciliaire : plate-forme d'échanges à la base des cils}

Dans la plupart des types cellulaires, la fusion de la vésicule ciliaire avec la membrane plasmique n'est pas suivie d'un relargage complet du cil à la surface. Le cil reste alors enraciné plus ou moins profondément dans le cytoplasme. Sa région proximale se retrouve entourée d'une invagination de la membrane plasmique. Ce domaine membranaire, à la forme caractéristique, a été observé dès la première description des cils primaires par B. Barnes en 1961 [2]. Hormis des observations réalisées dans les chondrocytes, qui ont suggéré son rôle potentiel dans le trafic vésiculaire [30], la présence de ce domaine à la base de la plupart des cils a cependant été progressivement oubliée (voir [31] pour revue).

Ce domaine membranaire a été récemment « redécouvert» par plusieurs groupes et appelé poche ciliaire en référence à la «poche flagellaire » du trypanosome, une structure à la base du flagelle responsable de tous les échanges membranaires chez cet organisme [31] (Figure 2). En effet, la poche ciliaire, présente à la base des différents types de cils (primaires, mobiles, flagelles) et dans de très nombreux types cellulaires différents (neurones, fibroblastes, chondrocytes), est également impliqué dans des évènements d'endocytose par la voie dépendante de la clathrine $[5,31]$. La poche ciliaire semble également se comporter comme un domaine d'interface entre le cil et le cytosquelette d'actine [5], qui a récemment été impliqué dans la régulation de la ciliogenèse $[23,32]$.

Beaucoup d'hypothèses ont été énoncées sur les fonctions possibles de la poche ciliaire. Le problème majeur reste l'absence d'outil permettant d'inhiber sa formation. Elle pourrait être impliquée dans des événements d'internalisation de protéines membranaires (récepteurs) provenant du cil et, ainsi, dans la régulation du signal après activation. Certains résultats vont dans ce sens : il a été montré que le cil primaire joue un rôle important dans la signalisation du récepteur du TGF $\beta$ (TGF $\beta$ R, transforming growth factor $\beta$ receptor), et que la poche ciliaire participerait à la régulation de ce récepteur via un cycle d'internalisation et un trafic vers une population d'endosomes proche de la base des cils. Ces évènements d'endocytose sont couplés au recrutement des voies de signalisation en aval du TGF $\beta R$ dans la région de la poche ciliaire [33]. La poche pourrait également jouer le rôle de plate-forme d'accueil pour des vésicules transportant des protéines membranaires et des récepteurs ciliaires provenant de la voie de biosynthèse (Golgi) ou des endosomes. Cette hypothèse est soutenue par une étude récente qui montre un phénomène de dilution des marqueurs de la membrane ciliaire lorsque l'on induit la formation de plusieurs cils dans des cellules et leur association à une même poche (comme si chaque poche était capable de prendre en charge une quantité finie de protéines membranaires ciliaires) [34].

\section{Conclusion}

L'étude des mécanismes responsables de la ciliogenèse montre les liens complexes qui existent entre le centrosome (les centrioles) et les membranes cellulaires, interactions dont certains acteurs moléculaires ont été récemment caractérisés. Ces liens semblent encore plus complexes qu'indiqués cidessus. Une très belle étude récente montre qu'au cours de la mitose, le cil est résorbé, mais le centriole père reste associé à une vésicule, «fantôme » de l'ancienne membrane ciliaire, tout au long de la division cellulaire. La cellule en division contient donc deux centrosomes dont un, le plus ancien, avec son centriole père préancré à une vésicule ciliaire. Celle des deux cellules filles recevant ce centrosome pourra alors former un cil plus rapidement et répondre ainsi à certains signaux avant sa sœur, ce qui représente une conséquence nouvelle et fascinante de la division asymétrique des centrosomes [35]. Les fonctions exercées par la poche ciliaire, quant à elles, restent mal caractérisées. Toutefois, la présence ou l'absence de cette poche permet d'identifier clairement deux types de cils. Elle est, par exemple, absente des cils impliqués dans la sensation de flux de liquides (tubules rénaux et canaux biliaires), mais présente, entre autres, à la base des cils des tissus conjonctifs (fibroblastes chondrocytes, ténocytes). Elle pourrait être impliquée à ce niveau dans l'orientation des cils au sein de ces tissus, et jouer ainsi un rôle important dans leurs fonctions mécanosensorielles (voir [5] pour détails). Pour tester ces hypothèses, il faudrait pouvoir inhiber la formation de cette poche sans interférer avec la ciliogenèse, ce qui permettrait de lever le mystère des fonctions exercées par cette structure étonnante associée à l'un des organites les plus fascinants du monde vivant. $\diamond$

\section{SUMMARY}

The ciliary pocket: a rendez-vous between the centrosome and vesicular trafficking

The assembly of cilia, ciliogenesis, involves complex and conserved mechanisms during which the basal body has to dock onto cell membranes. Studies in the $1960 \mathrm{~s}$ suggested that in many cell types such docking occurs in the cytoplasm and that cilia are formed within a vesicle before being delivered to the plasma membrane. This intracellular pathway, recently characterized at the molecular level, leads to the formation of a membrane domain at the basis of cilia, the ciliary pocket, which was involved in vesicular trafficking and signaling. $\diamond$

\section{LIENS D'INTÉRÊT}

L'auteur déclare n'avoir aucun lien d'intérêt concernant les données publiées dans cet article.

\section{REMERCIEMENTS}

Merci à Marion Failler pour sa lecture attentive de ce manuscrit et ses précieux conseils. Les travaux de recherche de l'auteur 
présentés dans cette revue ont été financés par l'Inserm, le CNRS, l'université Paris Descartes, le ministère de l'Éducation nationale et de la recherche scientifique, l'Agence nationale pour la recherche (ANR), la région ille-de-France (CORDDIM) et la Fondation pour la recherche médicale (FRM).

\section{RÉFÉRENCES}

1. Satir P, Christensen ST. Overview of structure and function of mammalian cilia. Annu Rev Physiol $2007 ; 69: 377-400$.

2. Barnes BG. Ciliated secretory cells in the pars distalis of the mouse hypophysis. J Ultrastruct Res $1961 ; 5: 453-67$.

3. Pazour GJ, Dickert BL, Vucica Y, et al. Chlamydomonas IFT88 and its mouse homologue, polycystic kidney disease gene $\operatorname{tg} 737$, are required for assembly of cilia and flagella.J Cell Biol 2000; 151 : 709-18.

4. Sorokin SP. Reconstructions of centriole formation and ciliogenesis in mammalian lungs. J Cell Sci $1968 ; 3: 207-30$.

5. Benmerah A. The ciliary pocket. Curr Opin Cell Biol $2013 ; 25: 78-84$.

6. Sorokin $\mathrm{S}$. Centrioles and the formation of rudimentary cilia by fibroblasts and smooth muscle cells. J Cell Biol $1962 ; 15: 363-77$.

7. Park TJ, Mitchell BJ, Abitua PB, et al. Dishevelled controls apical docking and planar polarization of basal bodies in ciliated epithelial cells. Nat Genet $2008 ; 40: 871-9$.

8. Anderson RG. The three-dimensional structure of the basal body from the rhesus monkey oviduct. J Cell Biol $1972 ; 54: 246-65$.

9. Ye X, Zeng H, Ning G, et al. C2cd3 is critical for centriolar distal appendage assembly and ciliary vesicle docking in mammals. Proc Natl Acad Sci USA $2014 ; 111: 2164-9$.

10. Tateishi $K$, Yamazaki Y, Nishida T, et al. Two appendages homologous between basal bodies and centrioles are formed using distinct Odf2 domains. J Cell Biol $2013 ; 203$ : 417-25.

11. Tanos BE, Yang H-J, Soni R, et al. Centriole distal appendages promote membrane docking, leading to cilia initiation. Genes Dev $2013 ; 27: 163-8$.

12. Graser S, Stierhof YD, Lavoie SB, et al. Cep164, a novel centriole appendage protein required for primary cilium formation. J Cell Biol $2007 ; 179$ : 321-30.

13. Joo K, Kim CG, Lee MS, et al. CCDC41 is required for ciliary vesicle docking to the mother centriole. Proc Natl Acad Sci USA 2013; 110 : 5987-92.

14. Sillibourne JE, Hurbain I, Grand-Perret T, et al. Primary ciliogenesis requires the distal appendage component Cep123. Biol Open 2013; 2 : 535-45.

15. Wei $Q, X u Q$, Zhang $Y$, et al. Transition fibre protein $F B F l$ is required for the ciliary entry of assembled intraflagellar transport complexes. Nat Commun $2013 ; 4: 2750$.

16. Chaki M, Airik R, Ghosh AK, et al. Exome capture reveals ZNF423 and CEP164 mutations, linking renal ciliopathies to DNA damage response signaling. Cell $2012 ; 150: 533-48$.

17. Adly N, Alhashem A, Ammari A, Alkuraya FS. Ciliary genes TBC1D32/C6orf170 and SCLT1 are mutated in patients with OFD type IX. Hum Mutat $2014 ; 35: 36-40$.

18. Failler M, Gee Hy, Krug P, et al. Mutations of CEP83 cause infantile nephronophthisis and intellectual disability. Am J Hum Genet 2014 ; 94 : 905-14.

19. Peränen J. Rab8 GTPase as a regulator of cell shape. Cytoskelet Hoboken NJ $2011 ; 68$ : 527-39.

20. Nachury MV, Loktev AV, Zhang $Q$, et al. A core complex of BBS proteins cooperates with the GTPase Rab8 to promote ciliary membrane biogenesis. Cell $2007 ; 129$ : 1201-13.

21. Schmidt KN, Kuhns S, Neuner A, et al. Cepl64 mediates vesicular docking to the mother centriole during early steps of ciliogenesis. J Cell Biol 2012 ; 199 : 1083-101.

22. Westlake CJ, Baye LM, Nachury MV, et al. Primary cilia membrane assembly is initiated by Rabll and transport protein particle II (TRAPPII) complex-dependent trafficking of Rabin8 to the centrosome. Proc Natl Acad Sci USA 2011 ; 108 : 2759-64.
23. Kim J, Lee JE, Heynen-Genel $S$, et al. Functional genomic screen for modulators of ciliogenesis and cilium length. Nature $2010 ; 464: 1048-51$.

24. Follit JA, Tuft RA, Fogarty KE, Pazour GJ. The intraflagellar transport protein IFT20 is associated with the Golgi complex and is required for cilia assembly. Mol Biol Cell 2006 ; 17 : 3781-92.

25. Finetti F, Paccani SR, Riparbelli MG, et al. Intraflagellar transport is required for polarized recycling of the TCR/CD3 complex to the immune synapse. Nat Cell Biol 2009; $11:$ 1332-9.

26. Omori Y, Zhao C, Saras A, et al. Elipsa is an early determinant of ciliogenesis that links the IFT particle to membrane-associated small GTPase Rab8. Nat Cell Biol $2008 ; 10: 437-44$.

27. Feng $S$, Knödler A, Ren J, et al. A Rab8 guanine nucleotide exchange factoreffector interaction network regulates primary ciliogenesis. J Biol Chem $2012 ; 287: 15602-9$

28. Zuo X, Guo W, Lipschutz JH. The exocyst protein Secl0 is necessary for primary ciliogenesis and cystogenesis in vitro. Mol Biol Cell $2009 ; 20$ : 2522-9.

29. Gonçalves J, Nolasco S, Nascimento R, et al. TBCCD1, a new centrosomal protein, is required for centrosome and Golgi apparatus positioning. EMBO Rep $2010 ; 11: 194-200$.

30. Poole CA, Flint MH, Beaumont BW. Analysis of the morphology and function of primary cilia in connective tissues: a cellular cybernetic probe? Cell Motil $1985 ; 5: 175-93$

31. Ghossoub R, Molla-Herman A, Bastin P, Benmerah A. The ciliary pocket: a once-forgotten membrane domain at the base of cilia. Biol Cell $2011 ; 103$ : 131-44.

32. Pitaval $A$, Tseng $Q$, Bornens $M$, Théry $M$. Cell shape and contractility regulate ciliogenesis in cell cycle-arrested cells. J Cell Biol $2010 ; 191: 303-12$.

33. Clement CA, Ajbro KD, Koefoed K, et al. TGF- signaling is associated with endocytosis at the pocket region of the primary cilium. Cell Rep $2013 ; 3$ : 1806-14.

34. Mahjoub MR, Stearns T. Supernumerary centrosomes nucleate extra cilia and compromise primary cilium signaling. Curr Biol CB 2012; 22 : 1628-34.

35. Paridaen JTML, Wilsch-Bräuninger M, Huttner WB. Asymmetric inheritance of centrosome-associated primary cilium membrane directs ciliogenesis after cell division. Cell $2013 ; 155: 333-44$

36. Fort $C$, Bastin $P$. Élongation de l'axonème et dynamique du transport intraflagellaire. Med Sci (Paris) 2014 ; 30 : 955-61.

37. Diguet N, Meilhac SM. Cils et morphogenèse cardiaque. Med Sci (Paris) $2014 ; 30: 996-1003$.

38. Ezan J, Montcouquiol M. Les liens multiples entre les cils et la polarité planaire cellulaire. Med Sci (Paris) 2014 ; 30 : 1004-10.

39. Paces-Fessy M. Cils et kystes rénaux. Med Sci (Paris) 2014 ; 30 : 1024-33.

40. Bachmann-Gagescu R. Complexité génétique des ciliopathies et identification de nouveaux gènes. Med Sci (Paris) $2014 ; 30$ : 1011-23.

41. Chennen K, Scerbo MJ, Dollfus H, et al. BBS : cils et obésité ; de la génétique à l'approche intégrative. Med Sci (Paris) 2014 ; 30 : 1034-9.

TIRÉS À PART

A. Benmerah

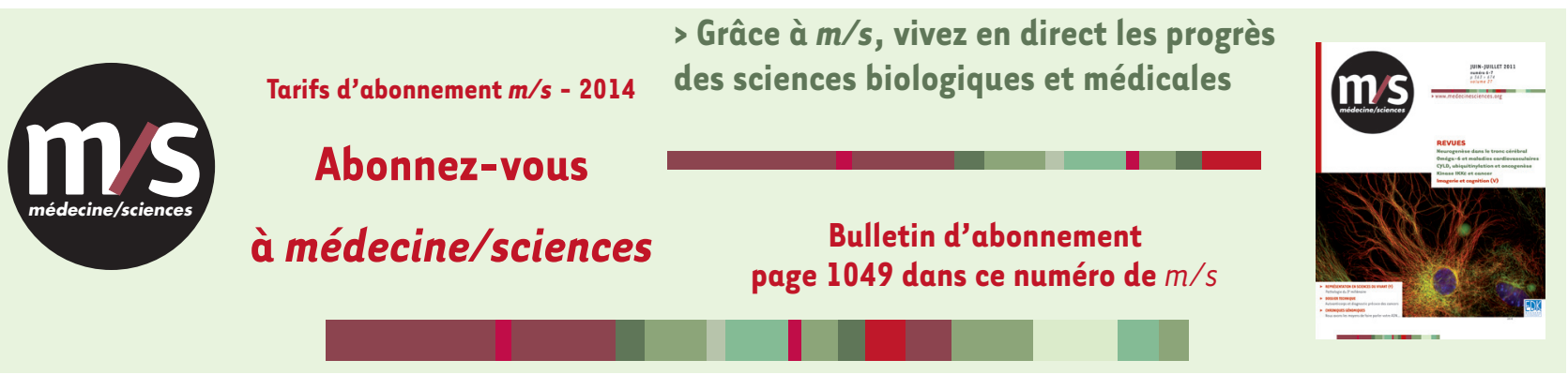

